\title{
Impulsividad y conciencia del problema predicen \\ la adherencia terapéutica y el abandono del tratamiento en el trastorno por juego de azar
}

\section{Impulsivity and problem awareness predict therapy compliance and dropout from treatment for gambling disorder}

\author{
María F. Jara-Rizzo ***, Juan F. Navas ****, Trevor Steward ****,*****, \\ Marta López-Gómez ******, Susana Jiménez-Murcia ******************, \\ Fernando Fernández-Aranda ****,*****,*******, José C. Perales *****. \\ * Centro de Investigación Mente, Cerebro y Comportamiento (CIMCYC). Universidad de Granada, Granada. España. ** \\ Universidad de Guayaquil, Guayaquil. Ecuador. *** Departamento de Psicología Experimental. Universidad de Granada, \\ Granada. España. **** Departamento de Psiquiatría. Hospital Universitario de Bellvitge-IDIBELL, Barcelona, España. ***** \\ Ciber Fisiopatología Obesidad y Nutrición (CIBERObn). Instituto de Salud Carlos III, Barcelona, España. ****** Asociación \\ Granadina de Jugadores de Azar en Rehabilitación (AGRAJER), Granada, España. ******* Departamento de Ciencias Clínicas. \\ Facultad de Medicina. Universidad de Barcelona, Barcelona, España.
}

\section{Resumen}

Este estudio investiga el valor predictivo de la impulsividad como rasgo (evaluada con la escala de conducta impulsiva UPPS-P) y de covariados relevantes (variables sociodemográficas, severidad del juego de azar, estado de ánimo disfórico, otras conductas adictivas e inteligencia no verbal), con respecto al abandono del tratamiento y los niveles de cumplimiento de las prescripciones terapéuticas en pacientes con trastorno por juego de azar. Sesenta y seis pacientes con este trastorno, participantes del proyecto G-Brain, fueron evaluados inicialmente en impulsividad rasgo y en los covariados mencionados. Dicha evaluación se realizó durante los seis primeros meses desde el inicio de su tratamiento. En el seguimiento realizado a los 6 meses, 24 pacientes habían abandonado (grupo ABD) y 42 continuaban el tratamiento (grupo NABD). Los análisis multivariados con las subescalas de impulsividad mostraron diferencias prospectivas entre ambos grupos. Aparentemente, estas diferencias son atribuibles a las dimensiones afectivas de impulsividad (urgencias positiva y negativa). Entre ambas dimensiones, solo la urgencia positiva fue un predictor independiente de un ligero incremento en la probabilidad de abandono. Dentro del grupo NABD, un mayor grado de adherencia terapéutica vino predicho, de manera independiente, tanto por una baja búsqueda de sensaciones como por una mayor conciencia de los problemas vinculados al juego. Estos resultados sugieren que los rasgos de impulsividad de origen afectivo son predictores de abandono del tratamiento en pacientes con trastorno por juego. La conciencia de problemas asociados al juego de azar y una baja búsqueda de sensaciones predisponen a una mayor adherencia a las prescripciones terapéuticas.

Palabras clave: Trastorno por juego de azar; Tratamiento; Impulsividad; Urgencia positiva; Búsqueda de sensaciones; Conciencia.

\begin{abstract}
This study investigates the predictive value of impulsivity traits (as measured by the UPPS-P impulsive behaviour scale) and relevant covariates (sociodemographics, gambling severity, dysphoric mood, other potentially addictive behaviours, and non-verbal intelligence) with regard to treatment dropout and level of adherence to therapy guidelines and instructions in patients with gambling disorder. Sixtysix patients seeking treatment for gambling disorder, and recruited to participate in a larger protocol (G-Brain), were initially assessed in impulsivity traits and relevant covariates in the first six months after admission. 24 patients dropped out (DO) and 42 patients remained in therapy (NDO) during the subsequent 6-month follow-up period. A multivariate analysis of impulsivity subscales suggested prospective differences between DO and NDO, with affect-driven dimensions (positive and negative urgency) seemingly driving these differences. Among these, only positive urgency independently predicted a slight increase in the drop-out probability. In the NDO group, a higher degree of adherence to therapy was independently predicted by lower sensation-seeking scores and stronger awareness of gambling-related problems. Results suggest the presence of affect-driven impulsivity traits as dropout predictors in patients with gambling disorder. Awareness of gambling-related problems and lower sensation-seeking enhanced compliance with therapeutic guidelines and instructions. Key words: Gambling disorder; Treatment; Impulsivity; Positive urgency; Sensation seeking; Awareness.
\end{abstract}

Recibido: Octubre 2017; Aceptado: Diciembre 2017

Enviar correspondencia a:

Juan F. Navas. Departamento de Psicología Experimental. Universidad de Granada. Campus de Cartuja, s/n 18071. Granada, España. E-mail: jfnavas@ugr.es 
$\mathrm{E}$ 1 trastorno por juego de azar (TJA) se caracteriza por la incapacidad para reducir o eliminar la participación excesiva en juegos de azar que conllevan apuestas monetarias, a pesar de la severidad de sus consecuencias negativas (American Psychiatric Association, 2013). Su prevalencia en las poblaciones adolescente y adulta varía entre el $0,4 \%$ y el 7,6\% (incluyendo tanto juego presencial como online, y dependiendo de la edad de la muestra, el tipo de herramientas y métodos, y lo estrictos que sean los criterios de significación clínica utilizados), con una prevalencia media del 2,2\% a nivel mundial (Jiménez-Murcia, Fernández-Aranda, Granero y Menchón, 2014). Los enfoques terapéuticos recomendados en la actualidad presentan una tasa de abandono prematuro del 30\%, aproximadamente (Aragay et al., 2015; Melville, Casey y Kavanagh, 2007).

De momento, el acuerdo es prácticamente unánime respecto de la consideración del TJA como una adicción comportamental (Leeman y Potenza, 2012; Petry, 2010); dicho consenso no se ha alcanzado respecto de otras supuestas adicciones comportamentales (Chacón-Cuberos et al., 2018; Martín-Fernández et al., 2017). No obstante, también hay evidencia de un alto nivel de variabilidad individual entre pacientes con TJA (Albein-Urios, Martinez-González, Lozano, Clark y Verdejo-García, 2012; Blaszczynski y Nower, 2002; Steward et al., 2017), que probablemente determine las respuestas diferenciales al tratamiento (Aragay et al., 2015; Chu y Clark, 2015; Melville et al., 2007)

\section{Impulsividad y curso del tratamiento del trastorno por juego de azar}

Varios estudios han intentado identificar los factores contextuales e individuales que pueden predecir los resultados del tratamiento. Por ejemplo, Weinstock et al. (2011) concluyeron que los factores sociodemográficos, la severidad del juego, el endeudamiento, y el nivel de coerción ejercido por parte de las redes legales y sociales son predictores de la aceptación o rechazo al tratamiento. Los patrones de juego (Pickering, Keen, Entwistle y Blaszczynski, 2018), las comorbilidades (Maniaci et al., 2017), y el apoyo interpersonal (Jiménez-Murcia et al., 2017) también han surgido como índices valiosos del curso del tratamiento y de sus resultados.

Nuestro estudio se centra en la personalidad impulsiva -la propensión a realizar actos de forma precipitada y sin meditación previa- y su valor potencial para predecir el abandono y la adherencia a las prescripciones terapéuticas durante el tratamiento. Otras evidencias relacionadas convergen en mostrar que: (1) las personas impulsivas son más propensas a desarrollar problemas relacionados con el juego en el futuro (Secades-Villa, Martínez-Loredo, Grande-Gosende, y Fernández-Hermida, 2016; van Holst, van den Brink, Veltman y Goudriaan, 2010; Vitaro, Brendgen, Ladouceur y Tremblay, 2001); (2) los pacientes con TJA con niveles altos de impulsividad tienen una mayor probabilidad de poner fin a su tratamiento de forma prematura (Leblond, Ladouceur y Blaszczynski, 2003; Maccallum, Blaszczynski, Ladouceur y Nower, 2007); (3) existe una asociación entre la impulsividad y una mayor comorbilidad psicopatológica, incluyendo otras adicciones (Grall-Bronnec et al., 2012; Petry, 2010); y (4) existe una correlación entre la impulsividad y la severidad del TJA (Billieux et al., 2012).

No obstante, la impulsividad se entiende mejor como un contructo multidimensional (Cyders y Smith, 2007; Evenden, 1999). Modelos factoriales recientes diferencian entre un factor de planificación-escrupulosidad que refleja la integridad de los mecanismos ejecutivos arriba-abajo, un factor de búsqueda de recompensa que se caracteriza por una sobrevaloración subjetiva de la recompensa (a pesar de las consecuencias negativas), y un factor de desregulación de las emociones negativas (Knezevic-Budisin, Pedden, White, Miller y Hoaken, 2015; Sharma, Markon y Clark, 2014).

Las herramientas psicométricas habituales se han basado en el análisis factorial de las respuestas a ítems de autoinforme, a la hora de identicar los componentes de la conducta impulsiva. En este sentido, el modelo UPPS-P del comportamiento impulsivo (Cyders et al., 2007; Whiteside, Lynam, Miller y Reynolds, 2001) ha mostrado ser más ventajoso que otras mediciones de la impulsividad, en términos de capacidad discriminativa y de correspondencia con sistemas psicobiológicos disociables (Rochat, Billieux, Gagnon y Van der Linden, 2017). A pesar de su valor teórico innegable, otros métodos de valoración alternativos basados en tareas neuropsicológicas o de toma de decisiones (por ejemplo, Torres et al., 2013a, 2013b), arrojan asociaciones muy modestas con herramientas de autoinforme, incluso para muestras muy grandes (Cyders et al., 2011; MacKillop et al., 2016). Hasta la fecha, no existe una batería de pruebas de este tipo que sea considerada como una alternativa comprehensiva y eficiente en términos de tiempo para evaluar la impulsividad como constructo multidimensional (Stahl et al., 2014).

Según el modelo UPPS-P, la impulsividad está compuesta de (1) urgencia positiva y (2) urgencia negativa, que representan tendencias a perder el control bajo emociones positivas y negativas, respectivamente; (3) falta de premeditación, que es la tendencia a tomar decisiones sin tener en cuenta sus consecuencias; (4) falta de perseverancia, que es la incapacidad de mantener el nivel de esfuerzo necesario durante una tarea exigente; y (5) búsqueda de sensaciones, que es la predisposición a probar actividades nuevas y estimulantes. Análisis de validez convergente sugieren que las urgencias responden a una combinación de reactividad emocional y desregulación ejecutiva; falta de premeditación y perseverancia se solapan, sobre todo, con el factor de planificación-escrupulosidad; la búsqueda de sensaciones revela una tendencia hacia la búsqueda de recompensas a través de actividades nuevas y estimulantes (Cyders y Smith, 2007; Sharma et al., 2014). 
De las dimensiones de la UPPS-P, la urgencia negativa emerge como el marcador más claro de severidad en niveles clínicos (Billieux et al., 2012), aunque también se ha observado que la urgencia positiva y la búsqueda de sensaciones predicen severidad en jugadores en tratamiento (Savvidou et al., 2017). En nuestro estudio, no obstante, exploramos la relación entre las dimensiones del UPPS-P y los resultados del tratamiento. Hasta donde sabemos, estudios previos no han analizado, desde una perspectiva multidimensional, la posible relación entre la impulsividad y la adherencia al tratamiento y/o el abandono del mismo.

A pesar de ello, algunas fuentes indirectas de evidencia nos permiten hacer ciertas predicciones sustantivas. Primero, al menos dos estudios han analizado la posible vinculación entre constructos que frecuentemente se solapan con la búsqueda de sensaciones y el abandono del tratamiento (Aragay et al., 2015; Jiménez-Murcia et al., 2012). Aunque dichos estudios no investigaron la impulsividad de forma directa, sí que evaluaron la personalidad mediante el cuestionario TCI (Temperament and Character Inventory, Cloninger, Svrakic y Przybeck, 1993). Ambos estudios hallaron que una de estas dimensiones (búsqueda de sensaciones) predecía el abandono. De manera análoga, otro estudio reciente (Mestre-Bach et al., 2016) mostró que las puntuaciones elevadas en un rasgo relacionado con la impulsividad y la búsqueda de sensaciones (la sensibilidad a la recompensa) se asociaba a una probabilidad más elevada de abandono del tratamiento, pero no con la severidad del trastorno, la ocurrencia de recaídas, o el cumplimiento del tratamiento. Dadas estas evidencias, la búsqueda de sensaciones de la UPPS-P parece un buen candidato como predictor del abandono.

Segundo, en vista del vínculo entre la urgencia negativa y las conductas adictivas, por vía de una regulación emocional alterada y habilidades de afrontamiento disfuncionales (Adams, Kaiser, Lynam, Charnigo y Milich, 2012), parece altamente plausible que la urgencia negativa contribuya de forma significativa a una adherencia insuficiente y al abandono.

\section{Objetivos e implicaciones clínicas}

Hasta la fecha, la mayoría de los estudios no han considerado medidas diferentes de la adherencia aparte de la continuidad del tratamiento, o no han hecho una diferenciación explícita entre el abandono y la adherencia (Melville et al., 2007). Aragay et al. (2015) no valoraron el cumplimiento de las prescripciones terapéuticas de forma explícita. Mestre-Bach et al. (2016) no tuvieron en cuenta el abandono al contabilizar el número de recaídas (por tanto, se confundían en cierta medida la recaída y el abandono), al tiempo que la adherencia se valoró de manera dicotómica (buena/mala). Hasta donde sabemos, solo Jiménez-Murcia et al. (2012) analizaron el cumpli- miento (mediante una escala de 3 puntos: bueno/razona$\mathrm{ble} / \mathrm{malo}$ ) de forma independiente del abandono. Como hemos dicho anteriormente, este estudio halló una asociación entre la búsqueda de sensaciones y el abandono, pero no se identificaron predictores significativos de adherencia al tratamiento.

Los objetivos del presente estudio fueron: (1) estimar hasta qué punto las dimensiones de la UPPS-P predicen el abandono del tratamiento psicológico (en los seis meses tras la valoración inicial), considerando varios confusores potenciales; y (2) poner a prueba si dichas variables, además, predicen el nivel de adherencia a las tareas y las recomendaciones terapéuticas, específicamente en los pacientes que continúan en tratamiento.

Potencialmente, los resultados tienen una relevancia clínica directa. Los cambios en el mercado de los juegos de azar, asociados a la aparición de nuevas modalidades de juego, suponen un gran desafío para los profesionales clínicos y los servicios de rehabilitación. Como hemos comentado anteriormente (Navas et al., 2017, Navas, Billieux, Verdejo-García, y Perales, 2018), los nuevos jugadores también presentan rasgos psicológicos específicos, y un paso necesario para personalizar el tratamiento es clarificar el valor pronóstico de dichos rasgos (Raylu y Oei, 2016).

\section{Método}

\section{Participantes}

En este estudio participaron 66 pacientes en tratamiento por TJA (2 mujeres) reclutados de la Asociación Granadina de Jugadores de Azar en Rehabilitación (AGRAJER), una asociación de apoyo mutuo ubicada en Granada, España. Como parte del protocolo de admisión, todos los pacientes participaron en una entrevista semi-estructurada con su terapeuta, basada en el DSM-IV para los trastornos de los ejes I y II, que incluía toda la información necesaria para verificar los criterios de exclusión. El diagnóstico de TJA fue realizado por el terapeuta a través de dicha entrevista, y fue además confirmado por una puntuación igual o superior a 5 en el cuestionario de juego patológico de South Oaks (SOGS, validación española; Echeburúa, Báez, Fernández-Montalvo y Páez, 1994).

Los criterios de inclusión fueron: (1) un diagnóstico de TJA, y (2) una duración del tratamiento menor a 6 meses completos. Los criterios de exclusión fueron: (1) comorbilidad con cualquier trastorno psiquiátrico del DSM-IV, y (2) cualquier historia de enfermedad neurológica o de daño cerebral (según autoinforme de los participantes). Otros pacientes del centro que potencialmente pudiesen sufrir un trastorno comórbido o que tuviesen una historia de daño neurológico no fueron invitados a participar en este estudio. Se aplicó el cuestionario MultiCAGE CAD-4 de evaluación clínica para detectar signos de uso problemático de alcohol o de drogas (Pedrero Pérez et al., 2007). 


\section{Procedimiento}

Evaluación inicial. La sesión de evaluación inicial duró aproximadamente tres horas. Aplicamos varios cuestionarios de autoinforme y pruebas neuropsicológicas, algunas de las cuales no son directamente relevantes para los fines de este estudio, ya que eran parte de un protocolo más amplio (proyecto G-Brain de investigación sobre el cerebro, PSI2013-45055-P), y han sido reportados anteriormente (por ejemplo, Navas et al., 2017; Navas, Verdejo-García, López-Gómez, Maldonado y Perales, 2016; Perales, Navas, Ruiz de Lara, Maldonado y Catena, 2017).

Es importante destacar que, dadas las características del centro de tratamiento y la disponibilidad limitada de pacientes, no siempre fue posible completar la valoración inicial de forma inmediata tras la admisión. En todos los casos, dicha evaluación inicial tuvo lugar durante los primeros seis meses de tratamiento. Concretamente, esta evaluación la completaron 22 pacientes en el primer mes, 20 en el segundo mes, 8 en el tercer mes, 3 en el cuarto mes, 7 en el quinto mes, y 6 en el sexto mes (la Tabla 1 muestra la duración media del tratamiento).

Seguimiento. Seis meses después de la evaluación inicial (y, por tanto, durante el primer año de tratamiento en todos los casos), se contactó con el terapeuta de los pacientes para recopilar información respecto del abandono/no abandono y de la adherencia al tratamiento (véase adherencia al tratamiento en el apartado Instrumentos). Según estos datos, la muestra original se dividió en dos grupos: 24 pacientes que abandonaron el tratamiento (ABD) y 42 pacientes que continuaron en él (NABD). La Tabla 1 muestra los estadísticos descriptivos de ambos grupos (panel superior).

\section{Instrumentos}

\section{Severidad del juego y otras conductas problemáticas}

Cuestionario de juego patológico de South Oaks (SOGS, Lesieur y Blume, 1987; versión española, Echeburúa et al., 1994). Este cuestionario de 20 ítems tiene como fin evaluar la severidad, el endeudamiento y la dependencia del juego. Tiene propiedades psicométricas adecuadas y es la herramienta más ampliamente usada en los estudios sobre TJA. En el presente estudio se utilizó únicamente la puntuación global de severidad.

MultiCAGE CAD-4 (Pedrero Pérez et al., 2007). Esta herramienta de cribado está compuesta por una serie de ítems dicotómicos para comprobar la presencia actual autopercibida de problemas relacionados con un control deficiente de los impulsos en varios ámbitos (incluyendo el juego y el uso de alcohol y de drogas ilegales). En el presente estudio se utilizaron únicamente las subescalas de juego, alcohol y drogas; todas han mostrado tener buenas propiedades psicométricas. Las demás subescalas (uso excesivo de internet y videojuegos, trastornos alimenticios, hipersexualidad, y compras compulsivas) no son relevantes para los fines del presente estudio.

Inteligencia no verbal estimada. El Cociente Intelectual (CI) no verbal se estimó usando la tarea de razonamiento de matrices de la Escala Wechsler de Inteligencia para Adultos (WAIS-IV; Wechsler, 2008).

Impulsividad. La escala breve de impulsividad UPPS-P (versión española, Cándido, Orduña, Perales, Verdejo-García y Billieux, 2012) está compuesta de 20 ítems tipo Likert dirigidos a valorar la urgencia negativa y urgencia positiva, la búsqueda de sensaciones, la falta de premeditación y la falta de perseverancia.

Estado de ánimo disfórico. Los signos subclínicos de estado de ánimo disfórico se evaluaron usando el Inventario de Depresión de Beck-II (BDI-II; versión española, Sanz, Perdigón y Vázquez, 2005). El cuestionario BDI-II se incluyó en el protocolo cuando algunos de los participantes ya habían sido evaluados. En consecuencia, faltan los datos del BDI para 4 pacientes, todos pertenecientes al grupo NABD.

Adherencia al tratamiento. La adherencia al tratamiento en pacientes NBAD se definió sobre la base de: (1) la asistencia a las actividades terapéuticas (e.g., sesiones grupales); y (2) la realización de tareas y cumplimiento de las directrices terapéuticas para el funcionamiento en la vida diaria (e.g., actualización de diarios, no consumo de alcohol). Sobre la base de los registros terapéuticos, todos los pacientes fueron evaluados en una escala de 5 puntos, en la cual 5 representaba asistencia y cumplimiento completa, 4 representaba asistencia y cumplimiento superior al 50\%; 3 representaba asistencia superior al $50 \%$ pero cumplimiento de tareas y directrices inferior al 50\%, 2 representaba asistencia y cumplimiento inferior al $50 \%$; y 1 representaba asistencia inferior al $50 \%$ y cumplimiento prácticamente nulo de tareas y directrices. Dos jueces independientes valoraron el nivel de cumplimiento (el segundo y cuarto autores), con una concordancia de $r=0,952$. Los casos de discrepancia en las valoraciones entre jueces se resolvieron por acuerdo mutuo. Entre los 42 pacientes que no abandonaron el tratamiento, 6 obtuvieron una puntuación de cinco, 13 de cuatro, 14 de tres, 7 de dos y 2 de un punto.

\section{Características del tratamiento}

Todos los participantes siguieron el mismo protocolo de tratamiento, con el mismo terapeuta, en las mismas instalaciones. Dicho tratamiento es similar al tratamiento implementado en otras asociaciones pertenecientes a la federación regional a la que pertenece AGRAJER [Federación Andaluza de Asociaciones de Jugadores de Azar en Rehabilitación (FAJER)]. Gran parte del tratamiento se basa en grupos de apoyo mutuo -complementado con supervisión profesional y terapia cognitiva-conductual individual- y tiene una duración aproximada de dos años. Las características y las fases del tratamiento están descritas en los materiales suplementarios S1. 


\section{Normas éticas}

El procedimiento de este estudio cumple las normas de la Declaración de Helsinki de 1975, revisadas en el 2008, y fue aprobado por el Comité de Ética de estudios humanos de la Universidad de Granada (España), como parte del proyecto de investigación PSI2013-45055-P. Todos los participantes fueron informados de los objetivos del estudio y firmaron un consentimiento.

\section{Análisis estadístico}

Análisis del abandono. Para describir las diferencias entre los grupos NABD y ABD, primero aplicamos pruebas t entre-grupos a las variables sociodemográficas y de control. Dicho análisis pretendía identificar posibles variables de confusión antes de analizar las diferencias entre los grupos en las medidas de impulsividad.

Segundo, realizamos análisis multivariante de varianza/ covarianza (MANOVA-MANCOVA) en las cinco subescalas del cuestionario UPPS-P. En caso de identificar potenciales variables de confusión, pretendíamos incluirlas como covariables en el MANCOVA. En caso de hallar un efecto multivariado significativo entre los grupos, se aplicaron posteriormente pruebas t, variable por variable, con el fin de identificar el origen del efecto multivariado global. Informamos de los valores $\mathrm{p}$ y factores de Bayes para todas las pruebas t.

Tercero, el análisis variable por variable se complementó con un análisis de regresión logística paso a paso, con la pertenencia al grupo como variable dependiente (NABD contra ABD) y las dimensiones de impulsividad como predictores. Dicho análisis se hizo para poner a prueba si alguna de las variables de impulsividad en las que pudiera haber diferencias entre ambos grupos predecía la pertenencia al grupo de manera independiente de las demás.

Análisis de cumplimiento terapéutico del grupo NABD. Cuarto, y exclusivamente para el grupo NABD, implementamos un análisis correlacional bivariado para estimar las relaciones entre las variables sociodemográficas/de control y la medida de adherencia. De nuevo, hicimos dichos análisis para identificar potenciales variables de confusión y tenerlas en cuenta en pasos posteriores.

Por último, las medidas de impulsividad, junto con las potenciales variables de confusión, se incluyeron en un análisis de regresión logística paso a paso de la medida de cumplimiento. Esto fue complementado por un análisis de regresión Bayesiano para identificar la combinación de factores más predictiva (incluyendo medidas de impulsividad y potenciales variables de confusión) y la contribución individual de cada factor.

Los análisis Bayesianos y las pruebas t simples se realizaron usando el paquete estadístico JASP (http://jasp-stats. org). Los análisis Bayesianos se realizaron con la configuración establecida por defecto en el software. Los análisis
MANOVA/MANCOVA y de regresión logística se hicieron con el paquete SPSS 20.0 (IBM Corp, 2011).

Dado que nuestra muestra tuvo únicamente a 2 mujeres participantes, todos los análisis se hicieron con y sin ellas. Puesto que los resultados fueron prácticamente idénticos en todos los casos, no encontramos motivo alguno para excluir a las mujeres. Así, se informa de los resultados correspondientes a la muestra completa.

\section{Resultados}

\section{Verificaciones de comparabilidad de grupos}

No se encontraron diferencias significativas entre los grupos $\mathrm{ABD}$ y NABD en variables sociodemográficas y de control (Tabla 1, panel superior). Es especialmente importante corroborar que ambos grupos tuvieron una buena coincidencia en duración de tratamiento en el momento de la valoración inicial. Dado que la valoración se demoró más para algunos participantes que para otros, una atrición desigual antes de la valoración inicial podía haber desequilibrado este variable a favor de uno de ambos grupos. El emparejamiento, por tanto, garantiza la comparabilidad entre grupos a pesar de las diferencias entre los individuos en el momento de la valoración inicial. Es más, los factores Bayesianos fueron inferiores a 1 de forma consistente para todas las potenciales variables de confusión, lo que indica un buen emparejamiento entre ambos grupos, en general.

Debido a algunas modificaciones menores en el formulario usado durante el estudio para recopilar los datos sociodemográficos, la edad de inicio en los juegos de azar estuvo disponible únicamente para 49 participantes. De estos, 17 pertenecían al grupo ABD y 32 al grupo NABD. Para estos dos subgrupos, no hubo una diferencia significativa en la edad de inicio [media (SD) 21,61 (7,69) y 19,47 $(5,72)$ para los subgrupos NABD y ABD, respectivamente, $\left.t(47)=1,01, p=0,32, \mathrm{BF}_{10}=0,45\right]$. De forma complementaria, disponíamos de datos sobre la modalidad de juego preferida (juegos de tipo I frente a tipo II, según la definición de Navas et al., 2017) para 65 participantes. Los resultados de las pruebas chi-cuadrado sobre la relación entre las preferencias y el abandono también fueron no significativas $\left[\chi^{2}(1)=1,475, p=0,225\right]$.

\section{Abandono}

Dada la ausencia de potenciales variables de confusión entre las variables consideradas, no se incluyeron covariables en el análisis posterior de las medidas de impulsividad. El análisis MANOVA correspondiente detectó un efecto principal multivariado de grupo [Wilks' $\lambda=0,823, p=0,035$, $\left.\eta_{p}^{2}=0,177\right]$. Las pruebas t variable por variable (Tabla 1 , panel inferior) mostraron diferencias significativas en urgencia positiva y en urgencia negativa entre ambos grupos. El modelo de regresión logística clasificó correctamente 
Impulsividad y conciencia del problema predicen la adherencia terapéutica y el abandono del tratamiento en el trastorno de juego de azar

Tabla 1. Pruebas t de muestras independientes y factores de Bayes para las variables sociodemográficas, de control y de impulsividad (UPPS-P).

\begin{tabular}{|c|c|c|c|c|c|}
\hline & NABD & ABD & & & \\
\hline & Media (SD) & Media (SD) & $\mathbf{t}$ & $p$ & $B F_{10}$ \\
\hline \multicolumn{6}{|c|}{ Variables sociodemográficas y de control } \\
\hline Edad & $37,67(11,33)$ & $33,92(10,46)$ & 1,330 & 0,188 & 0,546 \\
\hline Años de estudio & $12,86(4,55)$ & $12,42(2,38)$ & 0,440 & 0,661 & 0,283 \\
\hline Meses en tratamiento & $2,85(1,72)$ & $2,36(1,34)$ & 1,193 & 0,237 & 0,473 \\
\hline WAIS IV Razonamiento de matrices & $96,31(14,86)$ & $99,17(14,42)$ & $-0,759$ & 0,450 & 0,332 \\
\hline BDI Estado de ánimo disfórico & $9,92(8,62)$ & $12,13(8,48)$ & $-0,987$ & 0,328 & 0,398 \\
\hline SOGS Severidad & $10,43(3,26)$ & $10,38(3,54)$ & 0,062 & 0,950 & 0,261 \\
\hline MC Juego & $3,07(0,89)$ & $2,75(0,94)$ & 1,377 & 0,173 & 0,576 \\
\hline MC Alcohol & $1,14(1,37)$ & $0,79(1,22)$ & 1,042 & 0,301 & 0,411 \\
\hline MC Drogas & $0,52(1,04)$ & $0,42(0,93)$ & 0,418 & 0,678 & 0,280 \\
\hline \multicolumn{6}{|l|}{ UPPS-P } \\
\hline Urgencia negativa & $2,73(0,72)$ & $3,10(0,69)$ & $-2,047$ & 0,045 & 1,481 \\
\hline Urgencia positiva & $2,48(0,59)$ & $2,78(0,53)$ & $-2,061$ & 0,043 & 1,516 \\
\hline Búsqueda de sensaciones & $2,14(0,66)$ & $2,46(0,91)$ & $-1,647$ & 0,104 & 0,808 \\
\hline (Falta de) Premeditación & $2,19(0,73)$ & $2,28(0,64)$ & $-0,508$ & 0,614 & 0,290 \\
\hline (Falta de) Perseverancia & $1,97(0,68)$ & $1,81(0,64)$ & 0,929 & 0,356 & 0,374 \\
\hline
\end{tabular}

Nota. Abreviaturas: $\mathrm{NABD}=$ Grupo de no abandono; $\mathrm{ABD}=$ grupo de abandono; $M C=$ MultiCAGE CAD-4. Las pruebas significativas aparecen en negrita.

Tabla 2. Resultados del análisis de regresión logística paso a paso para pertenencia al grupo (no abandono [NABD] contra abandono $[A B D])$.

\begin{tabular}{|c|c|c|c|c|c|c|}
\hline $\begin{array}{c}\text { Variable } \\
\text { dependiente }\end{array}$ & $\begin{array}{l}\text { Variables } \\
\text { incluidas }\end{array}$ & $\begin{array}{l}\text { Variables } \\
\text { excluidas }\end{array}$ & $-2 \Delta L L$ & Wald & $p$ & $N-R^{2}$ \\
\hline \multirow[t]{2}{*}{$\begin{array}{c}\text { NABD } \\
\text { contra ABD }\end{array}$} & $\begin{array}{l}\text { Urgencia } \\
\text { positiva }\end{array}$ & & 4,285 & 3,882 & 0,049 & 0,086 \\
\hline & & $\begin{array}{c}\text { Urgencia } \\
\text { negativa } \\
\text { Búsqueda de } \\
\text { sensaciones } \\
\text { Falta de } \\
\text { premeditación } \\
\text { Falta de } \\
\text { perseverancia }\end{array}$ & & & & \\
\hline
\end{tabular}

Nota. Los valores $p$ para pruebas significativas aparecen en negrita. $-2 \Delta L L:-2$ log-verosimilitud diferencial para la inclusión de la urgencia negativa en el modelo; $N-R^{2}: R^{2}$ de Nagelkerke.
Tabla 3. Correlaciones del cumplimiento terapéutico con variables sociodemográficas y de control en el grupo de no abandono.

\begin{tabular}{lcc}
\hline & \multicolumn{2}{c}{ Cumplimiento del tratamiento } \\
& $\boldsymbol{r}$ & $\boldsymbol{p}$ \\
\hline Edad & 0,053 & 0,737 \\
Años de estudio & 0,175 & 0,269 \\
Edad de inicio de juego & 0,058 & 0,752 \\
Meses en tratamiento & 0,190 & 0,228 \\
Razonamiento de matrices (WAIS IV) & 0,392 & $\mathbf{0 , 0 1 0}$ \\
Estado de ánimo disfórico (BDI) & 0,366 & $\mathbf{0 , 0 2 4}$ \\
Severidad de juego (SOGS) & $-0,042$ & 0,792 \\
MC Juego & 0,482 & $\mathbf{0 , 0 0 1}$ \\
MC Alcohol & $-0,132$ & 0,403 \\
MC Drogas & $-0,007$ & 0,964 \\
\hline
\end{tabular}

Nota. Los valores $p$ para pruebas significativas aparecen en negrita. Abreviaturas: $M C=$ MultiCAGE CAD-4. Consulte el texto para información detallada de los instrumentos. La correlación entre la edad de inicio de juego y el cumplimiento terapéutico se hizo en 32 participantes del grupo NABD con estos datos disponibles.

anecdótica a favor de la hipótesis alternativa. Por tanto, los valores $\mathrm{p}$ significativos han de interpretarse con cautela, ya que meramente sugieren la ubicación específica de los efectos sobre la impulsividad en general, en caso de que hubiera alguno. 
Tabla 4. Resultados del análisis de regresión logística paso a paso sobre las puntuaciones en cumplimiento terapéutico para el grupo de no abandono.

\begin{tabular}{|c|c|c|c|c|c|}
\hline $\begin{array}{l}\text { Variables } \\
\text { incluidas }\end{array}$ & $\begin{array}{l}\text { Variables } \\
\text { excluidas }\end{array}$ & $\beta$ & $t$ & $p$ & Adj. $R^{2}$ \\
\hline MC Juego & & 0,465 & 3,311 & 0,002 & 0,272 \\
\hline \multirow{7}{*}{$\begin{array}{l}\text { Búsqueda de } \\
\text { sensaciones }\end{array}$} & & $-0,319$ & $-2,274$ & 0,029 & \\
\hline & $\begin{array}{c}\text { Razonamiento } \\
\text { de matrices }\end{array}$ & & & & \\
\hline & $\begin{array}{c}\text { Estado de ánimo } \\
\text { disfórico }\end{array}$ & & & & \\
\hline & $\begin{array}{l}\text { Urgencia } \\
\text { negativa }\end{array}$ & & & & \\
\hline & $\begin{array}{l}\text { Urgencia } \\
\text { positiva }\end{array}$ & & & & \\
\hline & $\begin{array}{c}\text { Falta de } \\
\text { premeditación }\end{array}$ & & & & \\
\hline & $\begin{array}{c}\text { Falta de } \\
\text { perseverancia }\end{array}$ & & & & \\
\hline
\end{tabular}

Nota. Los valores $p$ para pruebas significativas aparecen en negrita. MC: MultiCAGE CAD-4. Consulte el texto para información detallada de los instrumentos.

\section{Cumplimiento terapéutico}

En el análisis de correlación en el grupo NABD, hubo una correlación positiva con la puntuación en razonamiento de matrices de WAIS, la subescala de juegos de azar en el cuestionario MultiCAGE CAD-4 (MC Juego), y el BDI (Tabla 3). Las preferencias de juego (tipo I frente a tipo II) no tuvieron un impacto significativo sobre el cumplimiento $[t(63)=-0,63, p=0,532]$. Dicho de otra manera, los pacientes con mayores niveles de cumplimiento tenían mejor habilidad de razonamiento, peor estado de ánimo, y consideraban más problemática su práctica de juego.

El análisis de regresión lineal paso a paso, incluyendo estos tres factores y las puntuaciones de la UPPS-P como predictores, mostró efectos significativos para la búsqueda de sensaciones de la UPPS-P y la subescala MC Juego. Mientras que el primero predecía el cumplimiento de forma inversa, el segundo lo hacía de manera directa (Tabla 4). Debido a la pérdida de datos del BDI, tal y como se comentó anteriormente, estos análisis se hicieron sin 4 participantes. Por este motivo, repetimos el análisis sin las puntuaciones del BDI. Los resultados de este análisis fueron cualitativamente idénticos, pero notablemente más nítidos [ $R^{2}$ ajustado $=0,308, p<0,001$; MC Juego: $\beta=0,470$, $p<0,001$; Búsqueda de sensaciones: $\beta=-0,330, p=0,015]$.

Complementamos estos análisis de regresión con un modelo de regresión Bayesiano. Como muestra la Tabla 5, el modelo con el factor de Bayes más alto (31,73 en relación al modelo nulo), y que, por tanto, mejor explica los datos, incluyó la urgencia negativa y la búsqueda de sensaciones de la UPPS-P y la subescala MC Juego. No obstante, los tres factores contribuyeron de manera diferente al ajuste predictivo
Tabla 5. Resultados del análisis de regresión logística paso a paso Bayesiana sobre el cumplimiento terapéutico para el grupo de no abandono.

\begin{tabular}{lcccc}
\hline \multicolumn{1}{c}{ Modelos } & $\mathbf{P}(\mathbf{M})$ & $\mathbf{P}(\mathbf{M} \mid$ data $)$ & $\mathbf{B F}_{\mathbf{M}}$ & $\mathbf{B F 1 0}$ \\
\hline Nulo & 0,004 & $9,095 \cdot 10-4$ & 0,232 & 1 \\
$\begin{array}{l}\text { MC Juego + urgencia } \\
\text { negativa + búsqueda } \\
\text { de sensaciones }\end{array}$ & 0,004 & 0,029 & 7,576 & 31,725 \\
$\begin{array}{l}\text { MC Juego + Búsqueda } \\
\text { de sensaciones }\end{array}$ & 0,004 & 0,024 & 6,172 & 25,982 \\
$\begin{array}{l}\text { MC Juego } \\
\begin{array}{l}\text { MC Juego + Urgencia } \\
\text { negativa }\end{array}\end{array}$ & 0,004 & 0,006 & 1,591 & 6,817 \\
$\begin{array}{l}\text { Urgencia negativa } \\
\text { + Búsqueda de } \\
\text { sensaciones }\end{array}$ & 0,004 & 0,000582 & 0,148 & 0,64 \\
\hline
\end{tabular}

Nota. $\mathrm{P}(\mathrm{M})$ : probabilidad a priori de los modelos. $\mathrm{P}(\mathrm{M} \mid$ data): probabilidad a posteriori de los modelos con los datos disponibles. $\mathrm{BF}_{\mathrm{M}}$ : factor de Bayes del modelo. BF10: factor de Bayes del modelo, relativo al nulo. El modelo con mayor capacidad explicativa.

del modelo. El factor de Bayes del mejor modelo, en relación a los equivalentes sin cada uno de los tres factores, fue 1,22, 4,65, y 49,57, al omitir la urgencia negativa, la búsqueda de sensaciones, y MC Juego, respectivamente. Según el análisis de regresión estándar descrito anteriormente, mientras que los modelos con y sin la urgencia negativa rindieron casi igual de bien (con una contribución muy modesta de la urgencia negativa sobre el ajuste predictivo del modelo), las contribuciones de la búsqueda de sensaciones y de MC Juego fueron sustanciales y fuertes (según indican los factores de Bayes mayores de 3 y 10, respectivamente).

\section{Discusión}

Los estudios disponibles hasta la fecha han identificado un número de variables individuales que influyen en el riesgo de abandonar el tratamiento antes de su finalización (e.g., Ramos-Grille, Gomà-i-Freixanet, Aragay, Valero y Vallès, 2013), y también algunas características de la terapia que aumentan o disminuyen su eficacia clínica (e.g., Cowlishaw et al., 2012; Jiménez-Murcia et al., 2015). No obstante, hasta donde sabemos, ninguno de estos estudios ha implementado un análisis detallado de las diferentes dimensiones de la impulsividad como predictores de abandono o de la adherencia a la terapia, controlando, a la vez, por potenciales variables confusoras. Nuestros resultados amplían la evidencia de que los factores individuales son determinantes en la reacción de los pacientes al tratamiento (Billieux et al., 2012; Blaszczynski y Nower, 2002; Ledgerwood y Petry, 2006).

Primero, nuestros resultados sugieren que dimensiones de impulsividad de base afectiva discriminan entre pacien- 
tes que continúan o abandonan su terapia (ABD y NABD). No obstante, basándonos en las relaciones teóricas entre la urgencia negativa y los procesos básicos de regulación de las emociones (Billieux et al., 2012; Clark et al., 2012; Michalczuk, Bowden-Jones, Verdejo-Garcia y Clark, 2011), habíamos predicho que esta dimensión sería un predictor sustancial e independiente del abandono. Aunque hallamos evidencia que sugería que la urgencia negativa era más elevada en el grupo $\mathrm{ABD}$, dicho efecto se minimizó por la urgencia positiva.

Aragay et al. (2015) y Jiménez-Murcia et al. (2012) habían reportado que la búsqueda de novedad predecía el abandono. La medida de búsqueda de sensaciones utilizada en nuestro estudio -que se corresponde en parte con la anterior- no discriminó entre pacientes de los grupos ABD y NABD. No obstante, la búsqueda de sensaciones y la búsqueda de novedad no son constructos que se solapan en su totalidad (Cloninger, 1991; Cyders y Coskunpinar, 2011), y, lo que es aún más importante, la búsqueda de novedad y la urgencia positiva abarcan procesos motivacionales apetitivos similares. Esto es coherente con el vínculo entre el abandono y la dependencia de la recompensa que hemos observado en los tratamientos para otras adicciones (López-Torrecillas, Perales, Nieto-Ruiz y Verdejo-García, 2014)

En caso de que esto se confirme, la urgencia positiva podría tener una potencial superioridad predictiva con respecto a otras dimensiones de impulsividad relacionadas con la recompensa. Esta cuestión podría surgir del hecho de que la urgencia está más determinada por procesos ejecutivos y relacionados con el control (Billieux, Gay, Rochat y Van der Linden, 2010; Cyders y Smith, 2008; Dir, Karyadi y Cyders, 2013; Grall-Bronnec et al., 2012). De hecho, estudios recientes han identificado dos vías diferentes mediante las cuales la impulsividad podría tener un impacto sobre las conductas potencialmente adictivas. La primera implica la debilidad de los sistemas de autorregulación, y la segunda, la reacción exagerada de los sistemas afectivos automáticos (Lannoy, Billieux y Maurage, 2014). Nuestros resultados apuntan a que estas dos mismas vías podrían estar involucradas en el riesgo de abandono temprano en TJA. Tanto la motivación para seguir jugando (impulsada por las propiedades recompensantes de las actividades del juego) como la incapacidad para regular la conducta influenciada por las emociones generadas por dichos motivadores apetitivos podrían interferir con la motivación para continuar en tratamiento. En todo caso, es importante tener en cuenta que la diferencia entre los grupos en la urgencia positiva, aunque significativa, mostró evidencia escasa de un efecto real. Como hemos señalado anteriormente, cualquier interpretación de este efecto ha de hacerse con cautela.

Los resultados son más claros respecto del cumplimiento de las indicaciones terapéuticas en los pacientes que no abandonaron su tratamiento tras el periodo de seguimiento. Conjuntamente, una inteligencia más alta, un estado de ánimo más depresivo, un estado autopercibido de juego más severo, y una menor puntuación en la búsqueda de sensaciones tuvieron una correlación positiva con el cumplimiento de las instrucciones y los consejos terapéuticos. En otras palabras, aparentemente no solo las motivaciones apetitivas aumentan la probabilidad de abandonar la terapia, sino que también algún grado de disforia facilita la adherencia de los pacientes que no abandonan su tratamiento.

La búsqueda de sensaciones fue la única dimensión de la impulsividad que predijo la falta de cumplimiento y, de forma relativamente inesperada, unas puntuaciones más altas en el MultiCAGE CAD-4 incrementaban el cumplimiento de manera independiente. Tentativamente, esta relación puede explicarse por la percepción de las consecuencias negativas del juego excesivo. De hecho, los cuatro ítems del MultiCAGE CAD-4 relacionados con el juego evalúan la presencia de craving, sentir culpabilidad, admitir haber engañado a otras personas, y admitir problemas relacionados con la familia, el dinero o el empleo. $\mathrm{Al}$ menos tres de estos ítems pueden contribuir a una percepción aumentada de la inutilidad del juego (y la utilidad del tratamiento), especialmente teniendo en cuenta que el vínculo entre el MultiCAGE CAD-4 y el cumplimiento se halló únicamente en los casos menos complicados de pacientes que continuaron en terapia. Esta interpretación es congruente con informes anteriores en los cuales se sugiere que es más probable que los consumidores de drogas y alcohol busquen tratamiento cuando tienen puntuaciones más altas en el cuestionario CAGE de abuso de alcohol (el antecesor del MultiCAGE CAD-4: Mayfield, McLeod y Hall, 1974), y cuando perciben mayor severidad de sus problemas relacionados con las drogas, según las medidas inspiradas en el CAGE (Ferri, Gossop, Rabe-Hesketh y Laranjeira, 2002).

\section{Implicaciones clínicas}

Este estudio identifica dos posibles objetivos a considerar por parte de los terapeutas cuando tratan a pacientes con TJA. Primero, su incapacidad para gestionar las emociones, que aparentemente bloquea los esfuerzos terapéuticos, lo que implica que la regulación emocional debe abordarse durante las fases iniciales del tratamiento (Jiménez-Murcia et al., 2015). Segundo, la búsqueda de sensaciones podría interferir con los esfuerzos terapéuticos para lograr que los pacientes reconozcan las consecuencias negativas del juego. De hecho, algunos autores han propuesto que los factores motivacionales sesgan las cogniciones sobre el juego, como predicen los modelos de razonamiento motivado (Navas et al., 2016). Por tanto, la conciencia de problemas podría ser sensible a estrategias de entrenamiento en habilidades metacognitivas (Mansueto et al., 2016).

De manera relacionada, la aparición de nuevos tipos de jugadores implica un difícil reto para los servicios profesionales que dispensan tratamiento. Aspectos de la impulsividad relacionados con la recompensa y el afecto positivo 
(urgencia positiva y búsqueda de sensaciones) parecen prevalentes en los usuarios patológicos o de alto riesgo de nuevos dispositivos y plataformas de juego (Barrault y Varescon, 2016; Goldstein, Vilhena-Churchill, Stewart, Hoaken, y Flett, 2016). Nuestros resultados sugieren que es más probable que los tratamientos predominantes en la actualidad no tengan éxito con estos pacientes, y que la causa de dicho aumento del riesgo de fracaso es más fácilmente atribuible a las características psicológicas de los pacientes que a sus preferencias de juego per se.

Por último, este estudio también hace una llamada a la cautela por parte de los terapeutas que tratan a pacientes con TJA. El abandono temprano impide la disponibilidad de feedback sobre la eficacia del tratamiento en casos complicados, es decir, aquellos con peor habilidad de regulación emocional. Existe el riesgo de que el feedback recibido por el terapeuta sobre la eficacia del tratamiento esté inflado por la pérdida temprana de información (Einhorn y Hogarth, 1978), dado que los cambios pre-post terapia, atribuidos a la misma, no suelen tener en cuenta el abandono temprano.

\section{Limitaciones y fortalezas}

Este estudio presenta algunas limitaciones a tener en cuenta. Primero, la evaluación inicial no siempre se realizó de manera inmediata tras la admisión a terapia del paciente. Algunos de los pacientes estuvieron en tratamiento hasta seis meses antes de realizar la evaluación. Dicho retraso en valorar a algunos pacientes deja abierta la posibilidad de no haber detectado algunos de los abandonos y que, por tanto, fueran omitidos de este estudio. Aunque este hecho puede hasta cierto punto limitar la generalizabilidad, no había diferencia de duración de tratamiento entre los grupos ABD y NABD cuando participaron en dicha evaluación inicial. También es importante mencionar que la evaluación de seguimiento únicamente incluyó a los abandonos que ocurrieron durante el primer año de un protocolo de tratamiento de dos años. Esto podría implicar que las variables identificadas puedan ser predictoras de resultados relativamente tempranos, pero no de los más tardíos. En trabajos futuros (actualmente en curso), abordaremos los resultados relativos a fases más avanzadas del tratamiento.

La segunda limitación implica que los resultados no sean necesariamente generalizables a pacientes en otros tipos de terapia, dado que nuestros participantes recibieron un protocolo de tratamiento específico. En este caso, en tanto que la terapia fue impartida por una asociación de apoyo mutuo, introduce ciertas características (por ejemplo, la presencia de coterapeutas no profesionales, o la posibilidad de confrontación entre miembros de la asociación) que no se dan en otras formas más convencionales de terapia cognitiva-conductual.

Por último, el tamaño muestral estuvo limitado por el flujo admisión de nuevos pacientes en el centro de trata- miento donde se realizó el estudio, y que tuvo lugar durante un periodo temporal razonable. La falta de potencia estadística atribuible a las muestras podría haber causado que algunos predictores no alcanzasen la significación, especialmente en el análisis de regresión lineal restringido al grupo NABD.

A pesar de ello, este estudio tiene las siguientes fortalezas principales. Primero, el esfuerzo por controlar las variables sociodemográficas e intelectuales, habitualmente omitidas en estudios prospectivos. Segundo, la evaluación del cumplimiento de manera cuidadosa y quasi-cuantitativa, e independiente del abandono. Tercero, su relevancia clínica potencial.

\section{Financiación/Reconocimientos}

MFJR recibió financiación de un programa de becas ofrecida por la Universidad de Guayaquil en Ecuador, 2015 (Consejo de Educación Superior - CES). JCP y JFN recibieron apoyo del gobierno de España (Ministerio de Economía y Competitividad, Secretaría de Estado de Investigación, Desarrollo e Innovación; Convocatoria 2013 de Proyectos I + D de Excelencia), con número de referencia PSI201345055-P. JFN fue dotado de una ayuda para la investigación individual (Ministerio de Educación, Cultura y Deporte, Programa FPU, número de referencia FPU13/00669). JCP es miembro del grupo RETICS (RD12/0028/0017), financiada por el Ministerio de Sanidad y Consumo de España. TS recibió ayudas del Instituto de Salud Carlos III (FIS PI14/00290 y CIBERobn) y una ayuda parcial de los fondos FEDER -Una manera de hacer Europa-. CIBER Fisiopatología de la Obesidad y Nutrición (CIBERobn) es una iniciativa del ISCIII. SJM recibió una ayuda parcial de los fondos FEDER/Fondo Europeo de Desarrollo Regional -Una manera de hacer Europa- y una ayuda del Ministerio de Economía y Competitividad (PSI2015-68701-R).

\section{Conflicto de intereses}

Los autores declaran la inexistencia de conflictos de intereses.

\section{Referencias}

Adams, Z. W., Kaiser, A. J., Lynam, D. R., Charnigo, R. J. y Milich, R. (2012). Drinking motives as mediators of the impulsivity-substance use relation: Pathways for negative urgency, lack of premeditation, and sensation seeking. Addictive Behaviors, 37, 848-855. doi:10.1016/j. addbeh.2012.03.016.

Albein-Urios, N., Martinez-González, J. M., Lozano, O., Clark, L. y Verdejo-García, A. (2012). Comparison of impulsivity and working memory in cocaine addiction and pathological gambling: Implications for cocaine-in- 
duced neurotoxicity. Drug and Alcohol Dependence, 126, 1-6. doi:10.1016/j.drugalcdep.2012.03.008.

American Psychiatric Association. (2013). Diagnostic and statistical manual of mental disorders. 5 th edn. Washington, DC: APA.

Aragay, N., Jiménez-Murcia, S., Granero, R., Fernández-Aranda, F., Ramos-Grille, I., Cardona, S., ... Vallès, V. (2015). Pathological gambling: Understanding relapses and dropouts. Comprehensive Psychiatry, 57, 58-64. doi:10.1016/j.comppsych.2014.10.009.

Barrault, S. y Varescon, I. (2016). Online and live regular poker players: Do they differ in impulsive sensation seeking and gambling practice? Journal of Behavioral Addictions, 5, 41-50. doi:10.1556/2006.5.2016.015.

Billieux, J., Gay, P., Rochat, L. y Van der Linden, M. (2010). The role of urgency and its underlying psychological mechanisms in problematic behaviours. Behaviour Research and Therapy, 48, 1085-1096. doi:10.1016/j. brat.2010.07.008.

Billieux, J., Lagrange, G., Van der Linden, M., Lançon, C., Adida, M. y Jeanningros, R. (2012). Investigation of impulsivity in a sample of treatment-seeking pathological gamblers: A multidimensional perspective. Psychiatry Research, 198, 291-296. doi:10.1016/j.psychres.2012.01.001.

Blaszczynski, A. y Nower, L. (2002). A pathways model of problem and pathological gambling. Addiction, 97, 487499. doi:10.1046/j.1360-0443.2002.00015.x.

Cándido, A., Orduña, E., Perales, J. C., Verdejo-García, A. y Billieux, J. (2012). Validation of a short Spanish version of the UPPS-P impulsive behaviour scale. Trastornos Adictivos, 14, 73-78. doi:10.1016/S1575-0973(12) 70048-X.

Chacón Cuberos, R., Zurita Ortega, F., Castro Sánchez, M., Espejo Garcés, T., Martínez Martínez, A. y Ruiz-Rico Ruiz, G. (2018). Relación entre autoconcepto, consumo de sustancias y uso problemático de videojuegos en universitarios: un modelo de ecuaciones estructurales. Adicciones, 30, 179-188. doi:10.20882/adicciones.872.

Chu, S. y Clark, L. (2015). Cognitive and neurobiological aspects of problem gambling: Relevance to treatment. Canadian Journal of Addiction, 6, 62-71.

Clark, L., Stokes, P. R., Wu, K., Michalczuk, R., Benecke, A., Watson, B. J., ... Lingford-Hughes, A. R. (2012). Striatal dopamine D2/D3 receptor binding in pathological gambling is correlated with mood-related impulsivity. NeuroImage, 63, 40-46. doi:10.1016/j.neuroimage.2012.06.067.

Cloninger, C. R. (1991). The Tridimensional Personality Questionnaire: U.S. normative data. Psychological Reports, 69, 1047-1057. doi: 10.2466/PR0.69.7.1047-1057.

Cloninger, C., Svrakic, D. y Przybeck, T. (1993). A psychobiological model of temperament and character. Archives of General Psychiatry, 50, 975-990. doi:10.1001/ archpsyc.1993.01820240059008.

Cowlishaw, S., Merkouris, S., Dowling, N., Anderson, C., Jackson, A. y Thomas, S. (2012). Psychological the- rapies for pathological and problem gambling. Cochrane Database of Systematic Reviews, 11, CD008937. doi:10.1002/14651858.CD008937.pub2.

Cyders, M. A. y Smith, G. T. (2007). Mood-based rash action and its components: Positive and negative urgency. Personality and Individual Differences, 43, 839-850. doi:10.1016/j.paid.2007.02.008.

Cyders, M. A. y Smith, G. T. (2008). Emotion-based dispositions to rash action: positive and negative urgency. Psychological Bulletin, 134, 807-828. doi:10.1037/a0013341.

Cyders, M. A. y Coskunpinar, A. (2011). Measurement of constructs using self-report and behavioral lab tasks: Is there overlap in nomothetic span and construct representation for impulsivity? Clinical Psychology Review, 31, 965-982. doi:10.1016/j.cpr.2011.06.001.

Cyders, M. A, Smith, G. T., Spillane, N. S., Fischer, S., Annus, A. M. y Peterson, C. (2007). Integration of impulsivity and positive mood to predict risky behavior: Development and validation of a measure of positive urgency. Psychological Assessment, 19, 107-118. doi:10.1037/10403590.19.1.107.

Dir, A. L., Karyadi, K. y Cyders, M. A. (2013). The uniqueness of negative urgency as a common risk factor for selfharm behaviors, alcohol consumption, and eating problems. Addictive Behaviors, 38, 2158-2162. doi:10.1016/j. addbeh.2013.01.025.

Echeburúa, E., Báez, C., Fernández-Montalvo, J. y Páez, D. (1994). Cuestionario de juego patológico de South Oaks (SOGS): Validación Española. [The South Oaks Gambling Screen (SOGS); Spanish validation]. Análisis y Modificación de Conducta, 20, 769-791.

Einhorn, H. J. y Hogarth, R. M. (1978). Confidence in judgment: Persistence of the illusion of validity. Psychological Review, 85, 395-416. doi:10.1037/0033-295X.85.5.395

Evenden, J. L. (1999). Varieties of impulsivity. Psychopharmacology, 146, 348-361. doi:10.1007/PL00005481.

Ferri, C. P., Gossop, M., Rabe-Hesketh, S. y Laranjeira, R. R. (2002). Differences in factors associated with first treatment entry and treatment re-entry among cocaine users. Addiction, 97, 825-832. doi:10.1046/j.13600443.2002.00130.x.

Goldstein, A. L., Vilhena-Churchill, N., Stewart, S. H., Hoaken, P. N. y Flett, G. L. (2016). Mood, motives, and money: An examination of factors that differentiate online and non-online young adult gamblers. Journal of Behavioral Addictions, 5, 68-76. doi:10.1556/2006.5.2016.003.

Grall-Bronnec, M., Wainstein, L., Feuillet, F., Bouju, G., Rocher, B., Vénisse, J. L. Y Sébille-Rivain, V. (2012). Clinical profiles as a function of level and type of impulsivity in a sample group of at-risk and pathological gamblers seeking treatment. Journal of Gambling Studies, 28, 239-252. doi:10.1007/s10899-011-9258-9.

IBM Corp. (2011). IBM SPSS Statistics for Windows. Armonk, NY: IBM Corp. 
Jiménez-Murcia, S., Fernández-Aranda, F., Granero, R. y Menchón, J. M. (2014). Gambling in Spain: Update on experience, research and policy. Addiction, 109, 15951601. doi:10.1111/add.12232.

Jiménez-Murcia, S., Tremblay, J., Stinchfield, R., Granero, R., Fernández-Aranda, F., Mestre-Bach, G., ... Menchón, J. M. (2017). The involvement of a concerned significant other in gambling disorder treatment outcome. Journal of Gambling Studies, 33, 937-953. doi:10.1007/ s10899-016-9657-z.

Jiménez-Murcia S., Granero R., Fernández-Aranda F., Arcelus J., Aymamí M.N., Gómez-Peña M., ... Menchón, J. M. (2015). Predictors of outcome among pathological gamblers receiving cognitive behavioral group therapy. European Addiction Research, 21, 169-178. doi:10.1159/000369528.

Jimenez-Murcia, S., Aymamí, N., Gómez-Peña, M., Santamaría, J. J., Álvarez-Moya, E., Fernández-Aranda, F., ... Menchón, J. M. (2012). Does exposure and response prevention improve the results of group cognitive-behavioural therapy for male slot machine pathological gamblers? British Journal of Clinical Psychology, 51, 54-71. doi:10.1111/j.2044-8260.2011.02012.x.

Knezevic-Budisin, B., Pedden, V., White, A., Miller, C. J. y Hoaken, P. N. (2015). A Multifactorial Conceptualization of Impulsivity. Journal of Individual Differences, 36, 191-198. doi:10.1027/1614-0001/a000173.

Lannoy, S., Billieux, J. y Maurage, P. (2014). Beyond inhibition: A dual-process perspective to renew the exploration of binge drinking. Frontiers in Human Neuroscience, 8 , 405. doi:10.3389/fnhum.2014.00405.

Leblond, J., Ladouceur, R. y Blaszczynski, A. (2003). Which pathological gamblers will complete treatment? The British Journal of Clinical Psychology, 42, 205-209. doi:10.1348/014466503321903607.

Ledgerwood, D. M. y Petry, N. M. (2006). Psychological experience of gambling and subtypes of pathological gamblers. Psychiatry Research, 144, 17-27. doi:10.1016/j. psychres.2005.08.017.

Leeman, R. F. y Potenza, M. N. (2012). Similarities and differences between pathological gambling and substance use disorders: A focus on impulsivity and compulsivity. Psychopharmacology, 219, 469-490. doi:10.1007/s00213011-2550-7.

Lesieur, H. R. y Blume, S. B. (1987). The South Oaks Gambling Screen (SOGS): A new instrument for the identification of pathological gamblers. American Journal of Psychiatry, 144, 1184-1188. doi:10.1176/ajp.144.9.1184.

López-Torrecillas, F., Perales, J. C., Nieto-Ruiz, A. y Verdejo-García, A. (2014). Temperament and impulsivity predictors of smoking cessation outcomes. PloS One, 9, e112440. doi:10.1371/journal.pone.0112440.

Maccallum, F., Blaszczynski, A., Ladouceur, R. y Nower, L. (2007). Functional and dysfunctional impulsivity in pa- thological gambling. Personality and Individual Differences, 43, 1829-1838. doi:10.1016/j.paid.2007.06.002.

MacKillop, J., Weafer, J., Gray, J. C., Oshri, A., Palmer, A. y de Wit, H. (2016). The latent structure of impulsivity: Impulsive choice, impulsive action, and impulsive personality traits. Psychopharmacology, 233, 3361-3370. doi:10.1007/s00213-016-4372-0.

Maniaci, G., La Cascia, C., Picone, F., Lipari, A., Cannizzaro, C. y La Barbera, D. (2017). Predictors of early dropout in treatment for gambling disorder: The role of personality disorders and clinical syndromes. Psychiatry Research, 257, 540-545. doi:10.1016/j.psychres.2017.08.003.

Mansueto, G., Pennelli, M., De Palo, V., Monacis, L., Sinatra, M. y De Caro, M. F. (2016). The role of metacognition in pathological gambling: A mediation model. Journal of Gambling Studies, 32, 93-106. doi:10.1007/ s10899-014-9519-5.

Mayfield, D., McLeod, G. y Hall, P. (1974). The CAGE questionnaire: Validation of a new alcoholism screening instrument. American Journal of Psychiatry, 131, 1121-1123.

Melville, K. M., Casey, L. M. y Kavanagh, D. J. (2007). Psychological treatment dropout among pathological gamblers. Clinical Psychology Review, 27, 944-958. doi:10.1016/j.cpr.2007.02.004.

Martín-Fernández, M., Matalí, J. L., García-Sánchez, S., Pardo, M., Lleras, M. y Castellano-Tejedor, C. (2017). Adolescents with Internet Gaming Disorder (IGD): Profiles and treatment response. Adicciones, 29, 125-133. doi:10.20882/adicciones.890.

Mestre-Bach, G., Granero, R., Steward, T., Fernández-Aranda, F., Baño, M., Aymami, N., ... Jiménez-Murcia, S. (2016). Reward and punishment sensitivity in women with gambling disorder or compulsive buying: Implications in treatment outcome. Journal of Behavioral Addictions, 5, 658-665. doi:10.1556/2006.5.2016.074.

Michalczuk, R., Bowden-Jones, H., Verdejo-Garcia, A. y Clark, L. (2011). Impulsivity and cognitive distortions in pathological gamblers attending the UK National Problem Gambling Clinic: A preliminary report. Psychological Medicine, 41, 2625-2635. doi:10.1017/ S003329171100095X.

Navas, J. F., Verdejo-García, A., López-Gómez, M., Maldonado, A. y Perales, J. C. (2016). Gambling with rose-tinted glasses on: Use of emotion-regulation strategies correlates with dysfunctional cognitions in gambling disorder patients. Journal of Behavioral Addictions, 5, 271281. doi:10.1556/2006.5.2016.040.

Navas, J. F., Billieux, J., Perandrés-Gómez, A., López-Torrecillas, F., Candido, A. y Perales, J. C. (2017). Impulsivity traits and gambling cognitions associated with gambling preferences and clinical status. International Gambling Studies, 17, 102-124. doi:10.1080/14459795.2016.12757 39 . 
Navas, J. F., Billieux, J., Verdejo-García, A. y Perales, J. C. (2018). A neurocognitive approach to core components of gambling disorder: Implications for assessment, treatment and policy. In H. Bowden-Jones, C. Dickson, C. Dunand, y O. Simon (Eds.), Harm Reduction for Problem Gambling: A Public Health Approach. (Aceptado). Routledge (due by March, 2018).

Pedrero Pérez, E. J., Rodríguez Monje, M. T., Gallardo Alonso, F., Fernández Girón, M., Pérez López, M. y Chicharro Romero, J. (2007). Validación de un instrumento para la detección de trastornos de control de impulsos y adicciones: el MULTICAGE CAD-4. [Validation of a tool for screening of impulsive control disorders and addiction: MULTICAGE CAD-4]. Trastornos Adictivos, 9, 269-278. doi:10.1016/S1575-0973(07)75656-8.

Perales, J. C., Navas, J. F., Ruiz de Lara, C. M., Maldonado, A. y Catena, A. (2017). Causal learning in gambling disorder: Beyond the illusion of control. Journal of Gambling Studies. 33, 705-717. doi:10.1007/s10899-016-9634-6.

Petry, N. M. (2010). Pathological gambling and the DSM-V. International Gambling Studies, 10, 113-115. doi:10.1080/ 14459795.2010 .501086$.

Pickering, D., Keen, B., Entwistle, G. y Blaszczynski, A. (2018). Measuring treatment outcomes in gambling disorders: A systematic review. Addiction, 113, 411-426. doi:10.1111/add.13968.

Ramos-Grille, I., Gomà-i-Freixanet, M., Aragay, N., Valero, S. y Vallès, V. (2013). The role of personality in the prediction of treatment outcome in pathological gamblers: A follow-up study. Psychological Assessment, 25, 599-605. doi:10.1037/a0031930.

Raylu, N. y Oei, T. P. S. (2016). Treatment planning for problem gamblers. Australian Clinical Psychologists, 2, 3-14.

Rochat, L., Billieux, J., Gagnon, J. y Van der Linden, M. (2017). A multifactorial and integrative approach to impulsivity in neuropsychology: insights from the UPPS model of impulsivity. Journal of Clinical and Experimental Neuropsychology, 55, 1-17. doi:10.1080/13803395.2017.13 13393.

Sanz, J., Perdigón, A. L. y Vázquez, C. (2005). Adaptación española del Inventario de Depression de Beck-II (BDIII):3. Propiedades psicométricas en pacientes con trastornos psicológicos. [Spanish adaptation of the Beck Depression Inventory-II (BDI-II): 3. Psychometric features in patients with psychological disorders]. Clinica y Salud, 16, 121-142.

Savvidou, L. G., Fagundo, A. B., Fernández-Aranda, F., Granero, R., Claes, L., Mallorquí-Baqué, N.,... Jiménez-Murcia, S. (2017). Is gambling disorder associated with impulsivity traits measured by the UPPS-P and is this association moderated by sex and age? Comprehensive Psychiatry, 72, 106-113. doi:10.1016/j.comppsych.2016.10.005.
Secades-Villa, R., Martínez-Loredo, V., Grande-Gosende, A. y Fernández-Hermida, J. R. (2016). The relationship between impulsivity and problem gambling in adolescence. Frontiers in Psychology, 7, 1931. doi:10.3389/ fpsyg.2016.01931.

Steward, T., Mestre-Bach, G., Fernández-Aranda, F., Granero, R., Perales, J. C., Navas, J. F., ... Jiménez-Murcia, S. (2017). Delay discounting and impulsivity traits in young and older gambling disorder patients. Addictive Behaviors, 71, 96-103. doi:10.1016/j.addbeh.2017.03.001.

Sharma, L., Markon, K. E. y Clark, L. A. (2014). Toward a theory of distinct types of "impulsive" behaviors: A meta-analysis of self-report and behavioral measures. Psychological Bulletin, 140, 374-408. doi:10.1037/a0034418.

Stahl, C., Voss, A., Schmitz, F., Nuszbaum, M., Tüscher, O., Lieb, K. y Klauer, K. C. (2014). Behavioral components of impulsivity. Journal of Experimental Psychology: General, 143, 850-866. doi:10.1037/a0033981.

Torres, A., Catena, A., Megías, A., Maldonado, A., Cándido, A., Verdejo-García, A. y Perales, J. C. (2013). Emotional and non-emotional pathways to impulsive behavior and addiction. Frontiers in Human Neuroscience, 7, 43. doi:10.3389/fnhum.2013.00043.

Torres, A., Catena, A., Cándido, A., Maldonado, A., Megías, A. y Perales, J. C. (2013). Cocaine Dependent Individuals and Gamblers Present Different Associative Learning Anomalies in Feedback-Driven Decision Making: A Behavioral and ERP Study. Frontiers in Psychology, 4, 122. doi:10.3389/fpsyg.2013.00122.

van Holst, R. J., van den Brink, W., Veltman, D. J. y Goudriaan, A. E. (2010). Why gamblers fail to win: A review of cognitive and neuroimaging findings in pathological gambling. Neuroscience and Biobehavioral Reviews, 34, 87107. doi:10.1016/j.neubiorev.2009.07.007.

Vitaro, F., Brendgen, M., Ladouceur, R. y Tremblay, R. E. (2001). Gambling, delinquency, and drug use during adolescence: Mutual influences and common risk factors. Journal of Gambling Studies, 17, 171-190. doi:10.1023/A:1012201221601.

Wechsler, D. (2008). Wechsler adult intelligence scale - Fourth Edition (WAIS-IV). San Antonio. NCS Pearson.

Weinstock, J., Burton, S., Rash, C. J., Moran, S., Biller, W., Krudelbach, N., ... Morasco, B. J. (2011). Predictors of engaging in problem gambling treatment: Data from the West Virginia Problem Gamblers Help Network. Psychology of Addictive Behaviors, 25, 372-379. doi:10.1037/ a0023240.

Whiteside, S. P., Lynam, D. R., Miller, J. D. y Reynolds, S. K. (2001). Validation of the UPPS Impulsive Behavior Scale: A four factor model of impulsivity. European Journal of Personality, 19, 559-574 . doi:10.1002/per.556. 


\section{S1. Materiales suplementarios Características del tratamiento}

Todos los participantes siguieron el mismo protocolo de tratamiento, con el mismo terapeuta, en las mismas instalaciones. Dicho tratamiento es similar al tratamiento implementado por otras asociaciones pertenecientes a la Federación Andaluza de Asociaciones de Jugadores de Azar en Rehabilitación (FAJER). La duración media del programa de tratamiento íntegro es de aproximadamente dos años. Las técnicas específicas aplicadas en el programa se basan en el modelo cognitivo-conductual.

El protocolo de tratamiento está compuesto de 4 fases. En la primera (1 sesión, Pre-bienvenida), el paciente tiene un contacto inicial con la institución, y se programa la sesión de bienvenida. En la segunda fase (1 sesión, Bienveni$d a$ ), dos coterapeutas (un ex-jugador y un familiar) dan la bienvenida al paciente y le animan a entrar en tratamiento. En la tercera fase (autoayuda y apoyo mutuo), las sesiones son grupales, con etapas que comprenden un compromiso preliminar, el comienzo de la rehabilitación y la participación en la misma. Las sesiones de esta fase son semanales, dirigidas por ex-jugadores y supervisadas por un terapeuta profesional. En paralelo y de manera parcial, una cuarta etapa (psicoterapia individual) es ofrecida por un psicólogo clínico de AGRAJER. La intervención individual sigue una temática psicoeducativa, diseñada para valorar el progreso terapéutico del paciente, para que adquiera conciencia de su proceso adictivo y sus síntomas, aprenda estrategias de prevención contra la recaída, examine las distorsiones cognitivas vinculadas al juego, fortalezca su autoestima, habilidades sociales y asertividad; aprenda a gestionar su ira, autocontrol y resolución de problemas; y para fomentar actividades recompensantes. En general, los pacientes necesitan un año para progresar a través de estas tres etapas, según el cumplimiento de los objetivos.

Al finalizar esta etapa, los pacientes abstinentes que no han abandonado el grupo son dados de alta y comienzan una fase final de seguimiento. En esta fase, las reuniones de los pacientes son mensuales, con una duración de hora y media. Los pacientes pueden seguir participando en estas reuniones el tiempo que quieran, dado su objetivo de consolidar la abstinencia y de proporcionar de herramientas para gestionar situaciones de riesgo que pueden resultar en una recaída.

En este estudio, el reclutamiento y la evaluación inicial se realizaron durante la fase de bienvenida o al principio de la fase de autoayuda y apoyo mutuo. 